Preprint

UCRL-ID-147297-PT-2

\title{
Kull ALE: II. Grid Motion on Unstructured Arbitrary Polyhedral Meshes
}

\author{
P. Anninos
}

This article was submitted to High Energy Astrophysics Division of American Astronomical Society, April Convention 2002

Albuquerque, NM, April 20 - 23, 2002

U.S. Department of Energy

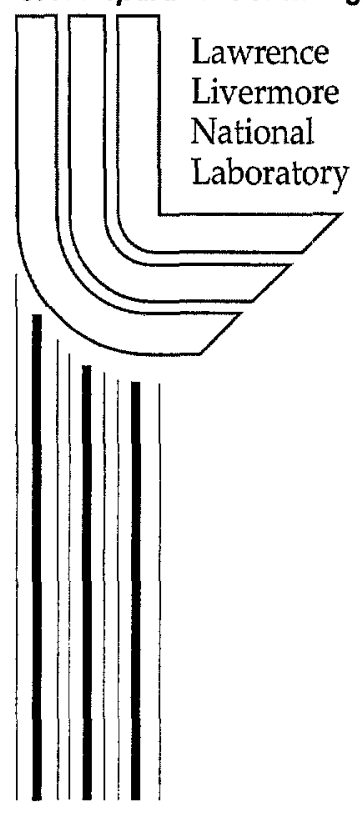

February 11, 2002 


\section{DISCLAIMER}

This document was prepared as an account of work sponsored by an agency of the United States Government. Neither the United States Government nor the University of California nor any of their employees, makes any warranty, express or implied, or assumes any legal liability or responsibility for the accuracy, completeness, or usefulness of any information, apparatus, product, or process disclosed, or represents that its use would not infringe privately owned rights. Reference herein to any specific commercial product, process, or service by trade name, trademark, manufacturer, or otherwise, does not necessarily constitute or imply its endorsement, recommendation, or favoring by the United States Government or the University of California. The views and opinions of authors expressed herein do not necessarily state or reflect those of the United States Government or the University of California, and shall not be used for advertising or product endorsement purposes.

This report has been reproduced directly from the best available copy.

Available electronically at http://www.doc.gov/bridge

Available for a processing fee to U.S. Department of Energy

And its contractors in paper from

U.S. Department of Energy

Office of Scientific and Technical Information

P.O. Box 62

Oak Ridge, TN 37831-0062

Telephone: (865) 576-8401

Facsimile: (865) 576-5728

E-mail: reports@adonis.osti.gov

Available for the sale to the public from

U.S. Department of Commerce

National Technical Information Service

5285 Port Royal Road

Springfield, VA 22161

Telephone: (800) 553-6847

Facsimile: (703) 605-6900

E-mail: orders@ntis.fedworld.gov

Online ordering: http://www.ntis.gov/ordering,htm

\section{OR}

Lawrence Livermore National Laboratory

Technical Information Department's Digital Library

http://www.llnl.gov/tid/Library.html 


\title{
Kull ALE: \\ II. Grid motion on unstructured arbitrary polyhedral meshes
}

\author{
Peter Anninos \\ University of California, \\ Lawrence Livermore National Laboratory, Livermore CA 94550
}

January 28,2002

\begin{abstract}
Several classes of mesh motion algorithms are presented for the remap phase of unstructured mesh ALE codes. The methods range from local shape optimization procedures to more complex variational minimization methods applied to arbitrary unstructured polyhedral meshes necessary for the Kull code.
\end{abstract}

\section{Local shape optimization}

Three different weighting schemes are described in this section to optimize the mesh configuration based on simple locally computed geometric shape functions. The first scheme attempts to adjust the vertex angles formed by the tetrahedral sub-zonal side elements to an optimal equi-angular state. The vertex solid angle $\left(\theta_{i}\right)$ is defined as the surface area of the tetrahedral side element $\mathrm{T}$ attached to the vertex $t_{i}$ and formed by projecting each point on the face not containing $t_{i}$ to the unit sphere centered on $t_{i}$. An explicit formula for $\theta_{0}$ at $t_{0}$ can be written in terms of the volume and edgelengths of T (Liu and Joe 1994)

$$
\sin \left(\theta_{0} / 2\right)=\frac{12 \mathrm{~V}}{\sqrt{\Pi_{1 \leq i \leq j \leq 3}\left(\ell_{0 i}+\ell_{0 j}+\ell_{i j}\right)\left(\ell_{0 i}+\ell_{0 j}-\ell_{i j}\right)}}
$$

where the volume is computed from the coordinates of each of the four vertices $\vec{x}_{i}$ at $t_{i}$

$$
V=\frac{1}{6} \operatorname{Det}\left(\begin{array}{cccc}
1 & x_{1} & y_{1} & z_{1} \\
1 & x_{2} & y_{2} & z_{2} \\
1 & x_{3} & y_{3} & z_{3} \\
1 & x_{4} & y_{4} & z_{4}
\end{array}\right)
$$

and $\ell_{i j} \equiv\left|\vec{\ell}_{i j}\right|$ are the edgelengths between vertices $t_{i}$ and $t_{j}$ with $\ell_{i j}=\left|t_{j}-t_{i}\right|$. The tetrahedron edges attached to $t_{0}$ are defined by the vectors connecting $t_{0}$ to the face centers $\left(t_{1}, \ell_{01}\right)$, the zone center $\left(t_{2}, \ell_{02}\right)$, and the opposite nodes $\left(t_{3}, \ell_{03}\right)$ of each of the $M$ sub-zonal side elements of the cell. The complete list of edges forming the tetrahedron are

$$
\begin{array}{lll}
\vec{\ell}_{01}=\vec{x}_{F}-\vec{x}_{0}, & \vec{\ell}_{02}=\vec{x}_{Z}-\vec{x}_{0}, & \vec{\ell}_{03}=\vec{x}_{N 1}-\vec{x}_{0}, \\
\vec{\ell}_{12}=\vec{x}_{Z}-\vec{x}_{F}, & \vec{\ell}_{13}=\vec{x}_{N 1}-\vec{x}_{F}, & \vec{\ell}_{23}=\vec{x}_{N 1}-\vec{x}_{Z} .
\end{array}
$$


By assigning an equilibrium angle appropriate for a regular tetrahedron, $\bar{\theta}=3 \cos ^{-1}(1 / 3)-\pi$, a weight factor is defined for the $m$ th side element as $W_{m}=\bar{\theta}-\theta_{0, m}$, to drive the nodal motion between pseudo-times $t^{n}$ and $t^{n+1}$ as

$$
\delta \vec{x}_{0}=\vec{x}_{0}^{n+1}-\vec{x}_{0}^{n}=\frac{\sum_{m=1}^{M}\left(\vec{x}_{F, m}^{n}+\vec{x}_{Z, m}^{n}+\vec{x}_{N 1, m}^{n}-3 \vec{x}_{0}^{n}\right) W_{m}}{3 \sum_{m=1}^{M}\left|W_{m}\right|} .
$$

In $2 \mathrm{D}$,

$$
\begin{aligned}
\theta_{0} & =\cos ^{-1}\left(\frac{\ell_{02}^{2}+\ell_{03}^{2}-\ell_{23}^{2}}{2 \ell_{02} \ell_{03}}\right) \\
\delta \vec{x}_{0} & =\frac{\sum_{m=1}^{M}\left(\vec{x}_{Z, m}+\vec{x}_{N 1, m}-2 \vec{x}_{0}\right) W_{m}}{2 \sum_{m=1}^{M} W_{m}}
\end{aligned}
$$

and $W_{m}=\pi / 3-\theta_{0, m}$.

A similar mesh calculation can be performed using the radius (or aspect) ratio defined by $\rho=r_{i} / r_{c}$, where $r_{i}$ and $r_{c}$ are the inscribed and circumscribed radii respectively. A convenient formula for computing $\rho$ is

$$
\rho=\frac{72 V^{2}}{\sum_{i=0}^{3} s_{i} \sqrt{(\alpha+\beta+\gamma)(\alpha+\beta-\gamma)(\alpha+\gamma-\beta)(\beta+\gamma-\alpha)}}
$$

where $\alpha=\ell_{01} \ell_{23}, \beta=\ell_{02} \ell_{13}$, and $\gamma=\ell_{03} \ell_{12}$ are products of the lengths of opposite edges of $\mathbf{T}$, and $s_{i}$ are the areas of the four faces

$$
s_{i}=\frac{1}{4} \sqrt{\left(a_{i}+b_{i}+c_{i}\right)\left(a_{i}+b_{i}-c_{i}\right)\left(a_{i}+c_{i}-b_{i}\right)\left(b_{i}+c_{i}-a_{i}\right)},
$$

with magnitudes of the three unique planar edge lengths making up the faces $a_{i}, b_{i}$ and $c_{i}$. Setting $W_{m}=\rho_{m}$ in (1.5) effectively drives the mesh to equal aspect ratio zoning. In $2 \mathrm{D}$, the corresponding shape parameter can be written

$$
\sigma=\frac{\left(\ell_{02}+\ell_{03}-\ell_{23}\right)\left(\ell_{23}+\ell_{03}-\ell_{02}\right)\left(\ell_{23}+\ell_{02}-\ell_{03}\right)}{\ell_{02} \ell_{03} \ell_{23}} .
$$

A more generic and fundamental representation of shape parameters is afforded by composing the Jacobian matrix of all node positions attached directly to $t_{0}$ (Freitag and Knupp 1999). Defining $\delta \vec{x}_{m, k}=\vec{x}_{m, k}-\vec{x}_{0}$ as the difference between the position of the $k$ th neighbor node of the $m$ th element and the position of the center node, the Jacobian matrix of the $m$ th element is written in terms of the relative vector components

$$
A_{m}=\left(\begin{array}{lll}
\delta x_{m, 1} & \delta x_{m, 2} & \delta x_{m, 3} \\
\delta y_{m, 1} & \delta y_{m, 2} & \delta y_{m, 3} \\
\delta z_{m, 1} & \delta z_{m, 2} & \delta z_{m, 3}
\end{array}\right)
$$

For 3D hexagonal cells or tetrahedral sub-zonal elements, $1 \leq k \leq 3$, and if an element is inverted or tangled, the determinant of the Jacobian matrix is Det $\left|A_{m}\right| \leq 0$. Defining the Frobenius norm

$$
|A|=\left[\operatorname{trace}\left(A^{T} A\right)\right]^{1 / 2}
$$


a variety of smoothness objective shape functions $f\left(A_{m}(\vec{x})\right)$ can be defined as criteria or weights for optimizing the mesh shape: $f\left(A_{m}(\vec{x})\right)=\left|A_{m}\right|^{2}, \operatorname{Det}\left|A_{m}\right|^{2}$, $\operatorname{Det}\left|A_{m}\right|^{2}\left|A_{m}^{-1}\right|^{2},\left|A_{m}\right|^{2} / \operatorname{Det}\left|A_{m}\right|$, interpreted as the sum of the square of the attached edge-lengths, the local cell volume, the sum of attached face areas squared, and the aspect ratio, respectively. The displacement vector in this case is defined by

$$
\delta \vec{x}_{0}=\frac{1}{\sum_{m=1}^{M}\left|W_{m}\right|} \sum_{m=1}^{M} \frac{\left(\ell_{01, m}^{n} \vec{\ell}_{01, m}^{n}+\ell_{02, m}^{n} \vec{\ell}_{02, m}^{n}+\ell_{03, m}^{n} \vec{\ell}_{03, m}^{n}\right) W_{m}}{\ell_{01, m}^{n}+\ell_{02, m}^{n}+\ell_{03, m}^{n}},
$$

where $W_{m}=f\left(A_{m}(\vec{x})\right)$.

\section{Tetrahedral finite element smoothing}

The focus in this section is on Laplacian smoothing on a tetrahedral finite element mesh which satisfies the condition of extremum in the variation integral

$$
I=\frac{1}{2} \int d x^{3} W(x) \nabla_{i} f \nabla^{i} f
$$

where $f$ is associated with the coordinates $(x, y, z)$, and $W(x)$ is an arbitrary weight function. Since polyhedral cells can be constructed out of more basic tetrahedral element, this approach is also relevant for more arbitrary polyhedral meshes.

The finite element approach presented here is based on the shape function of the sub-zonal tetrahedron (or triangle in 2D) side element composing of two nodes along an edge of the cell, one of the face centers, and the zone center. Using the notation $(\xi, \eta, \zeta) \rightarrow(r, s, t)$ for the generalized coordinates, the shape functions for an orthogonal tetrahedral element is defined by

$$
N_{1}=r, \quad N_{2}=s, \quad N_{3}=t, \quad \text { and } \quad N_{4}=1-r-s-t,
$$

where node $N_{4}$ corresponds to the pivotal node $t_{0}$ in the notation of section $\S 1$. Using the notation $\partial_{s} N \equiv \partial N / \partial s$, the shape function gradients take the form

$$
\vec{\nabla} N_{\alpha}=\left(\begin{array}{l}
\partial_{r} N_{\alpha} \partial_{x} r+\partial_{s} N_{\alpha} \partial_{x} s+\partial_{t} N_{\alpha} \partial_{x} t \\
\partial_{r} N_{\alpha} \partial_{y} r+\partial_{s} N_{\alpha} \partial_{y} s+\partial_{t} N_{\alpha} \partial_{y} t \\
\partial_{r} N_{\alpha} \partial_{z} r+\partial_{s} N_{\alpha} \partial_{z} s+\partial_{t} N_{\alpha} \partial_{z} t
\end{array}\right)
$$

which reduces to

$$
\begin{aligned}
& \vec{\nabla} N_{1}=\left(\begin{array}{c}
\partial_{x} r \\
\partial_{y} r \\
\partial_{z} r
\end{array}\right)=\frac{1}{J}\left(\begin{array}{ll}
\partial_{s} y \partial_{t} z-\partial_{t} y \partial_{s} z \\
\partial_{s} z \partial_{t} x-\partial_{t} z \partial_{s} x \\
\partial_{s} x \partial_{t} y-\partial_{t} x \partial_{s} y
\end{array}\right)=\frac{1}{J}\left(\begin{array}{c}
2 A_{1} \\
0 \\
0
\end{array}\right) \\
& \vec{\nabla} N_{2}=\left(\begin{array}{c}
\partial_{x} s \\
\partial_{y} s \\
\partial_{z} s
\end{array}\right)=\frac{1}{J}\left(\begin{array}{ll}
\partial_{t} y \partial_{r} z-\partial_{r} y \partial_{t} z \\
\partial_{t} z \partial_{r} x-\partial_{r} z \partial_{t} x \\
\partial_{t} x \partial_{r} y-\partial_{r} x \partial_{t} y
\end{array}\right)=\frac{1}{J}\left(\begin{array}{c}
0 \\
2 A_{2} \\
0
\end{array}\right) \\
& \vec{\nabla} N_{3}=\left(\begin{array}{c}
\partial_{x} t \\
\partial_{y} t \\
\partial_{z} t
\end{array}\right)=\frac{1}{J}\left(\begin{array}{l}
\partial_{r} y \partial_{s} z-\partial_{s} y \partial_{r} z \\
\partial_{r} z \partial_{s} x-\partial_{s} z \partial_{r} x \\
\partial_{r} x \partial_{s} y-\partial_{s} x \partial_{r} y
\end{array}\right)=\frac{1}{J}\left(\begin{array}{c}
0 \\
0 \\
2 A_{3}
\end{array}\right)
\end{aligned}
$$


and

$$
\vec{\nabla} N_{4}=-\left(\vec{\nabla} N_{1}+\vec{\nabla} N_{2}+\vec{\nabla} N_{3}\right)=-\frac{2}{J}\left(\begin{array}{c}
A_{1} \\
A_{2} \\
A_{3}
\end{array}\right) .
$$

$A_{i}$ are the areas of the opposite face (to the node $i$ ) triangles

$$
A=\frac{1}{2} \operatorname{Det}\left(\begin{array}{lll}
1 & x_{1} & y_{1} \\
1 & x_{2} & y_{2} \\
1 & x_{3} & y_{3}
\end{array}\right)
$$

and $J$ is the determinant of the Jacobian matrix related to the tetrahedral volume $V$

$$
J=\frac{\partial x}{\partial r}\left(\frac{\partial y}{\partial s} \frac{\partial z}{\partial t}-\frac{\partial y}{\partial t} \frac{\partial z}{\partial s}\right)+\frac{\partial x}{\partial s}\left(\frac{\partial y}{\partial t} \frac{\partial z}{\partial r}-\frac{\partial y}{\partial r} \frac{\partial z}{\partial t}\right)+\frac{\partial x}{\partial t}\left(\frac{\partial y}{\partial r} \frac{\partial z}{\partial s}-\frac{\partial y}{\partial s} \frac{\partial z}{\partial r}\right)=6 V
$$

Equations (2.17) - (2.19) can be written collectively in compact form as

$$
\vec{\nabla} N_{\alpha}=\frac{2 \vec{A}_{\alpha}}{J}=\frac{\vec{A}_{\alpha}}{3 V}
$$

where $\vec{A}_{\alpha}$ is inward pointing (towards the cell center).

Since an arbitrary function $f$ is represented in a finite element approach as $f=\sum_{\alpha} f_{\alpha} N_{\alpha}$, where $f_{\alpha}$ and $N_{\alpha}$ are the values of $f$ and the shape function at the $\alpha$ th grid point, the gradient of $f$ can be expanded as

$$
\vec{\nabla} f=\sum_{\alpha} f_{\alpha} \vec{\nabla} N_{\alpha}
$$

Associating $f$ with the coordinates $f \equiv(x, y, z)$ yields for the coordinates

$$
\begin{aligned}
& f \quad \equiv x=r x_{1}+s x_{2}+t x_{3}+(1-r-s-t) x_{4}, \\
& f \quad \equiv y=r y_{1}+s y_{2}+t y_{3}+(1-r-s-t) y_{4}, \\
& f \quad \equiv z=r z_{1}+s z_{2}+t z_{3}+(1-r-s-t) z_{4} .
\end{aligned}
$$

Substituting (2.24) into the variational integral (2.14), yields

$$
I=\frac{1}{2} \sum_{\alpha, \beta} f_{\alpha} f_{\beta} \int d x^{3} W \nabla_{i} N_{\alpha} \nabla^{i} N_{\beta}=\frac{1}{2} \sum_{\alpha, \beta} f_{\alpha} f_{\beta} A_{\alpha \beta}
$$

where

$$
A_{\alpha \beta}=\int d x^{3} W \nabla_{i} N_{\alpha} \nabla^{i} N_{\beta}=\int d x^{3} \frac{4 W}{J^{2}} \vec{A}_{\alpha} \cdot \vec{A}_{\beta},
$$

is the matrix composed of the inner vector products of opposite face areas (2.23). However, one drawback of this form is the tendency to drive the mesh to a state of orthogonal faces since $\vec{A}_{\alpha} \cdot \vec{A}_{\beta}=0$ represents the extremum. A particular manifestation of this problem appears in spherically converging meshes. In this case the mesh tends to concentrate radially towards the middle domains at which $r \sim \Delta r / \Delta \theta$, where the arc length $s=r \Delta \theta$ is comparable to the radial distance $\Delta r$ between nodes, and the side tetrahedral elements are naturally formed with minimal aspect ratios. This can be compensated for to some extent by adding an additional term to (2.28) 
to drive the system to a competing equilibrium state. This is acheived easily by considering the sum of the square of the edgelengths, and rewriting (2.28) as

$$
I=\frac{1}{2} \sum_{\alpha, \beta} f_{\alpha} f_{\beta} \int d x^{3} W \nabla_{i} N_{\alpha} \nabla^{i} N_{\beta}+\frac{\epsilon}{2} \sum_{\alpha} W \ell_{\alpha}^{2} V^{1 / 3},
$$

where $\epsilon$ is a coupling constant, $\ell_{\alpha}^{2}$ are the edgelengths squared, and the volume $V^{1 / 3}$ is introduced for dimensional reasons. A useful identity

$$
\sum_{\alpha} \ell_{\alpha}^{2}=\sum_{\alpha, \beta}\left(4 \delta_{\alpha}^{\beta}-1\right) f_{\alpha} f_{\beta}
$$

where $\delta_{\alpha}^{\beta}$ is the Kronecker symbol, can be substituted in (2.30) to yield

$$
I=\frac{1}{2} \sum_{\alpha, \beta} f_{\alpha} f_{\beta}\left(\frac{4 W}{J^{2}} \vec{A}_{\alpha} \cdot \vec{A}_{\beta}+\epsilon W V^{1 / 3}\left(4 \delta_{\alpha \beta}-1\right)\right)=\frac{1}{2} \sum_{\alpha, \beta} f_{\alpha} f_{\beta} A_{\alpha \beta},
$$

where now

$$
A_{\alpha \beta}=\int d x^{3} \frac{4 W}{J^{2}} \vec{A}_{\alpha} \cdot \vec{A}_{\beta}+\epsilon W V^{1 / 3}\left(4 \delta_{\alpha \beta}-1\right),
$$

and for a single element, ignoring overall constants

$$
A_{\alpha \beta}=\frac{W}{J}\left(\vec{A}_{\alpha} \cdot \vec{A}_{\beta}+\epsilon V^{4 / 3}\left(4 \delta_{\alpha \beta}-1\right)\right)
$$

A similar calculation can be carried out in two dimensions to yield

$$
A_{\alpha \beta}=\frac{W}{J}\left(\vec{S}_{\alpha} \cdot \vec{S}_{\beta}+\epsilon A\left(3 \delta_{\alpha \beta}-1\right)\right)
$$

where $\vec{S}_{\alpha}$ are the inward pointing opposite edge lengths, and $A$ is the area of the triangular cell.

Forcing the variational integral to extremum

$$
\frac{\partial I}{\partial f_{\alpha}}=\sum_{\beta} f_{\beta} A_{\alpha \beta}=0
$$

and introducing $\tilde{W}_{\beta}$ for anisotropic weighting yields

$$
\begin{aligned}
f_{\alpha}^{\ell} & =-\frac{1}{A_{\alpha \alpha}} \sum_{\beta \neq \alpha} A_{\alpha \beta} f_{\beta}, \\
& =-\frac{\tilde{W}^{\ell}}{\left|A_{\alpha}\right|^{2}+3 \epsilon V^{4 / 3}} \sum_{\beta \neq \alpha}\left(\vec{A}_{\alpha} \cdot \vec{A}_{\beta}+\epsilon V^{4 / 3}\left(4 \delta_{\alpha \beta}-1\right)\right) f_{\beta} .
\end{aligned}
$$

for the contribution from a single element $\ell$ to the modified coordinates of the $\alpha$ th node. 


\section{Generalized Laplacian smoothing on polyhedral meshes}

The variational approach discussed in section $\S 2$ is extended here to a weighted Winslow-Crowley (Crowley 1962; Winslow 1963) form on a single finite element and generalized to arbitrary polyhedrals. Using a general coordinate transformation between reference coordinates $\xi_{i}$ and Cartesian coordinates $x_{i}$ with $x_{i}=f_{i}\left(\xi_{j}\right)$, the variation integral (2.14) becomes (Tipton 1994)

$$
\begin{aligned}
I & =\frac{1}{2} \int d x^{3} W(x) \nabla_{i} f \nabla^{i} f \\
& =\frac{1}{2} \int d \xi^{3} J(\xi) W(\xi) \sum_{i, j, k} \frac{\partial \xi_{j}}{\partial x_{i}} \frac{\partial f}{\partial \xi_{j}} \frac{\partial \xi_{k}}{\partial x_{i}} \frac{\partial f}{\partial \xi_{k}} \\
& =\frac{1}{2} \int d \xi^{3} J(\xi) W(\xi) \sum_{i, j}\left(g_{i j}\right)^{-1} \frac{\partial f}{\partial \xi_{i}} \frac{\partial f}{\partial \xi_{j}}
\end{aligned}
$$

where the coordinate transformation is implicit in conventional tensor notation

$$
\left(g_{i j}\right)^{-1}=\sum_{k} \frac{\partial \xi_{i}}{\partial x_{k}} \frac{\partial \xi_{j}}{\partial x_{k}}
$$

Variation of $(3.41), \delta I=0$, gives

$$
\sum_{i j} \frac{\partial}{\partial \xi_{i}}\left(J W\left(g_{i j}\right)^{-1} \frac{\partial f}{\partial \xi_{j}}\right)=0
$$

and neglecting metric derivatives results in the Winslow-Crowley formula

$$
\sum_{i, j} J^{2}\left(g_{i j}\right)^{-1} \frac{\partial}{\partial \xi_{i}}\left(\frac{W}{J} \frac{\partial f}{\partial \xi_{j}}\right)=0
$$

assuming also that the weight factor is set to the Jacobian $W=J$.

In finite element form, the action is written

$$
I=\frac{1}{2} \sum_{\alpha, \beta} f_{\alpha} f_{\beta} \int d x^{3} W(x) \nabla_{i} N_{\alpha} \nabla^{i} N_{\beta}=\frac{1}{2} \sum_{\alpha, \beta} f_{\alpha} f_{\beta} A_{\alpha \beta}
$$

where the diffusion matrix is defined

$$
A_{\alpha \beta}=\int d x^{3} W(x) \nabla_{i} N_{\alpha} \nabla^{i} N_{\beta}=\int d \xi^{3} J(x) W(x) \sum_{i, j}\left(g_{i j}\right)^{-1} \frac{\partial N_{\alpha}}{\partial \xi_{i}} \frac{\partial N_{\beta}}{\partial \xi_{j}}
$$

and the new coordinates are computed by

$$
f_{\alpha}=-\frac{1}{A_{\alpha \alpha}} \sum_{\beta \neq \alpha} A_{\alpha \beta} f_{\beta}
$$

as in $\S 2$.

The difference between the tetrahedral results of the previous section and those discussed here lies primarily in the definition of the gradients used in (3.46). The notion of generalized coordinate 
transformations is applicable locally to a single (quadrilateral or hexagonal) element, though it may not necessarily be parametrically continuous across different elements. For a single element, the metric tensor representing the coordinate transformation is written as

$$
\begin{aligned}
\left(g_{i j}\right)^{-1} & =\sum_{k} \frac{\partial \xi_{i}}{\partial x_{k}} \frac{\partial \xi_{j}}{\partial x_{k}} \\
& =\frac{1}{J^{2}}\left(\begin{array}{lll}
g_{22} g_{33}-g_{23} g_{23} & g_{13} g_{23}-g_{12} g_{33} & g_{12} g_{23}-g_{22} g_{13} \\
g_{13} g_{23}-g_{12} g_{33} & g_{11} g_{33}-g_{13} g_{13} & g_{13} g_{12}-g_{11} g_{32} \\
g_{12} g_{23}-g_{22} g_{13} & g_{13} g_{12}-g_{11} g_{32} & g_{11} g_{22}-g_{12} g_{12}
\end{array}\right)
\end{aligned}
$$

where

$$
g_{i j}=\sum_{k} \frac{\partial x_{i}}{\partial \xi_{k}} \frac{\partial x_{j}}{\partial \xi_{k}}
$$

is the symmetric inverse of (3.49), with independent components

$$
\begin{aligned}
g_{11} & =\frac{\partial x}{\partial \xi} \frac{\partial x}{\partial \xi}+\frac{\partial y}{\partial \xi} \frac{\partial y}{\partial \xi}+\frac{\partial z}{\partial \xi} \frac{\partial z}{\partial \xi} \\
g_{12} & =\frac{\partial x}{\partial \xi} \frac{\partial x}{\partial \eta}+\frac{\partial y}{\partial \xi} \frac{\partial y}{\partial \eta}+\frac{\partial z}{\partial \xi} \frac{\partial z}{\partial \eta} \\
g_{13} & =\frac{\partial x}{\partial \xi} \frac{\partial x}{\partial \zeta}+\frac{\partial y}{\partial \xi} \frac{\partial y}{\partial \zeta}+\frac{\partial z}{\partial \xi} \frac{\partial z}{\partial \zeta} \\
g_{22} & =\frac{\partial x}{\partial \eta} \frac{\partial x}{\partial \eta}+\frac{\partial y}{\partial \eta} \frac{\partial y}{\partial \eta}+\frac{\partial z}{\partial \eta} \frac{\partial z}{\partial \eta} \\
g_{23} & =\frac{\partial x}{\partial \eta} \frac{\partial x}{\partial \zeta}+\frac{\partial y}{\partial \eta} \frac{\partial y}{\partial \zeta}+\frac{\partial z}{\partial \eta} \frac{\partial z}{\partial \zeta} \\
g_{33} & =\frac{\partial x}{\partial \zeta} \frac{\partial x}{\partial \zeta}+\frac{\partial y}{\partial \zeta} \frac{\partial y}{\partial \zeta}+\frac{\partial z}{\partial \zeta} \frac{\partial z}{\partial \zeta}
\end{aligned}
$$

and Jacobian determinant

$$
J=\frac{\partial x}{\partial \xi}\left(\frac{\partial y}{\partial \eta} \frac{\partial z}{\partial \zeta}-\frac{\partial y}{\partial \zeta} \frac{\partial z}{\partial \eta}\right)+\frac{\partial x}{\partial \eta}\left(\frac{\partial y}{\partial \xi} \frac{\partial z}{\partial \zeta}-\frac{\partial y}{\partial \zeta} \frac{\partial z}{\partial \xi}\right)+\frac{\partial x}{\partial \zeta}\left(\frac{\partial y}{\partial \xi} \frac{\partial z}{\partial \eta}-\frac{\partial y}{\partial \eta} \frac{\partial z}{\partial \xi}\right) .
$$

The Winslow-Crowley formula is readily generalized to an individual element and to account for metric differences across elements by including the metric derivatives and discretizing the full variational result (3.43) in place of (3.44). Discretizing (3.43), summing over all elements $\ell$, and treating the weight and metric variations in an explicit first order fashion gives

$$
\begin{aligned}
N f_{i, j, k} & =\sum_{\ell}\left[\left(g_{11}^{-1} J W\right)_{i+1 / 2, j, k}^{\ell} f_{i+1, j, k}+\left(g_{11}^{-1} J W\right)_{i-1 / 2, j, k}^{\ell} f_{i-1, j, k}\right] \\
& +\sum_{\ell}\left[\left(g_{22}^{-1} J W\right)_{i, j+1 / 2, k}^{\ell} f_{i, j+1, k}+\left(g_{22}^{-1} J W\right)_{i, j-1 / 2, k}^{\ell} f_{i, j-1, k}\right] \\
& +\sum_{\ell}\left[\left(g_{33}^{-1} J W\right)_{i, j, k+1 / 2}^{\ell} f_{i, j, k+1}+\left(g_{33}^{-1} J W\right)_{i, j, k-1 / 2}^{\ell} f_{i, j, k-1}\right] \\
& +\frac{1}{4} \sum_{\ell}\left[\left(g_{12}^{-1} J W\right)_{i+1 / 2, j+1 / 2, k}^{\ell} f_{i+1, j+1, k}+\left(g_{12}^{-1} J W\right)_{i-1 / 2, j-1 / 2, k}^{\ell} f_{i-1, j-1, k}\right]
\end{aligned}
$$




$$
\begin{aligned}
& -\frac{1}{4} \sum_{\ell}\left[\left(g_{12}^{-1} J W\right)_{i+1 / 2, j-1 / 2, k}^{\ell} f_{i+1, j-1, k}+\left(g_{12}^{-1} J W\right)_{i-1 / 2, j+1 / 2, k}^{\ell} f_{i-1, j+1, k}\right] \\
& +\frac{1}{4} \sum_{\ell}\left[\left(g_{13}^{-1} J W\right)_{i+1 / 2, j, k+1 / 2}^{\ell} f_{i+1, j, k+1}+\left(g_{13}^{-1} J W\right)_{i-1 / 2, j, k-1 / 2}^{\ell} f_{i-1, j, k-1}\right] \\
& -\frac{1}{4} \sum_{\ell}\left[\left(g_{13}^{-1} J W\right)_{i+1 / 2, j, k-1 / 2}^{\ell} f_{i+1, j, k-1}+\left(g_{13}^{-1} J W\right)_{i-1 / 2, j, k+1 / 2}^{\ell} f_{i-1, j, k+1}\right] \\
& +\frac{1}{4} \sum_{\ell}\left[\left(g_{23}^{-1} J W\right)_{i, j+1 / 2, k+1 / 2}^{\ell} f_{i, j+1, k+1}+\left(g_{23}^{-1} J W\right)_{i, j-1 / 2, k-1 / 2}^{\ell} f_{i, j-1, k-1}\right] \\
& \left.-\frac{1}{4} \sum_{\ell}\left[\left(g_{23}^{-1} J W\right)_{i, j-1 / 2, k+1 / 2}^{\ell} f_{i, j-1, k+1}+\left(g_{23}^{-1} J W\right)_{i, j+1 / 2, k-1 / 2}^{\ell} f_{i, j+1, k-1}\right] 3.58\right)
\end{aligned}
$$

for the coordinates of the central pivotal node. Also,

$$
\begin{aligned}
N & =\sum_{\ell}\left[\left(g_{11}^{-1} J W\right)_{i+1 / 2, j, k}^{\ell}+\left(g_{11}^{-1} J W\right)_{i-1 / 2, j, k}^{\ell}+\left(g_{22}^{-1} J W\right)_{i, j+1 / 2, k}^{\ell}\right] \\
& +\sum_{\ell}\left[\left(g_{22}^{-1} J W\right)_{i, j-1 / 2, k}^{\ell}+\left(g_{33}^{-1} J W\right)_{i, j, k+1 / 2}^{\ell}+\left(g_{33}^{-1} J W\right)_{i, j, k-1 / 2}^{\ell}\right]
\end{aligned}
$$

is the normalization factor, and the spatial indices on the terms $\left(g_{i j}^{-1} J W\right)^{\ell}$ indicate the edge alignment within element $\ell$ from which the metric components, Jacobian, and weight functions are derived.

The physical interpretation of the components of $g_{i j}$ implicit in the definition of $\left(g_{i j}^{-1}\right)$ is understood as scalar products of different permutations of any two edge vectors centered on an arbitrary pivotal node. In particular, $g_{i j}$ on node $\alpha$ is associated with the scalar product of the two edgelengths denoted by $(i, j)$ belonging to the same discrete element $\ell, g_{i j}=\delta \vec{x}_{(i)} \cdot \delta \vec{x}_{(j)}$. With this notation, the tensor components of (3.49) are written in the form

$$
\begin{aligned}
& J^{2}\left(g_{11}\right)^{-1}=\delta x_{k}^{(2)} \delta x_{(2)}^{k} \delta x_{l}^{(3)} \delta x_{(3)}^{l}-\left(\delta x_{k}^{(2)} \delta x_{(3)}^{k}\right)^{2} \\
& J^{2}\left(g_{12}\right)^{-1}=\delta x_{k}^{(1)} \delta x_{(3)}^{k} \delta x_{l}^{(2)} \delta x_{(3)}^{l}-\delta x_{k}^{(1)} \delta x_{(2)}^{k} \delta x_{l}^{(3)} \delta x_{(3)}^{l}, \\
& J^{2}\left(g_{13}\right)^{-1}=\delta x_{k}^{(1)} \delta x_{(2)}^{k} \delta x_{l}^{(2)} \delta x_{(3)}^{l}-\delta x_{k}^{(2)} \delta x_{(2)}^{k} \delta x_{l}^{(1)} \delta x_{(3)}^{l} \\
& J^{2}\left(g_{22}\right)^{-1}=\delta x_{k}^{(1)} \delta x_{(1)}^{k} \delta x_{l}^{(3)} \delta x_{(3)}^{l}-\left(\delta x_{k}^{(1)} \delta x_{(3)}^{k}\right)^{2} \\
& J^{2}\left(g_{23}\right)^{-1}=\delta x_{k}^{(1)} \delta x_{(3)}^{k} \delta x_{l}^{(1)} \delta x_{(2)}^{l}-\delta x_{k}^{(1)} \delta x_{(1)}^{k} \delta x_{l}^{(2)} \delta x_{(3)}^{l} \\
& J^{2}\left(g_{33}\right)^{-1}=\delta x_{k}^{(1)} \delta x_{(1)}^{k} \delta x_{l}^{(2)} \delta x_{(2)}^{l}-\left(\delta x_{k}^{(1)} \delta x_{(2)}^{k}\right)^{2}
\end{aligned}
$$

with an implied summation convention over identical covariant and contravariant indices.

Although derived assuming hexagonally shaped cells, the identification of the metric tensor with local edge products is easily extended to arbitrary polyhedrals by accounting for all possible permutations of edge pairings. For example, considering differential changes in nodal positions (as apposed to absolute coordinate positions), the node-centered net displacement function between 
$(69 \cdot \varepsilon)$

$$
\frac{l+g+o}{\partial^{\prime} 0 x \rho l+Z^{\prime} 0 x \rho g+N^{6} 0 x \rho D}=0 x \rho
$$

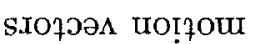

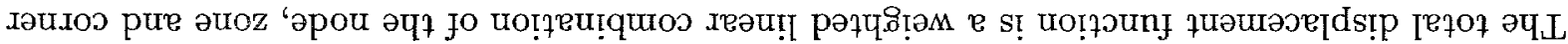

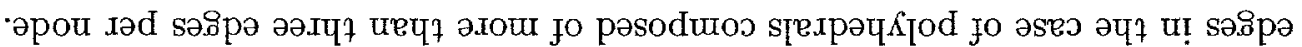

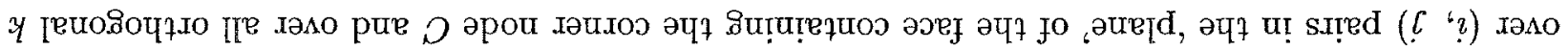

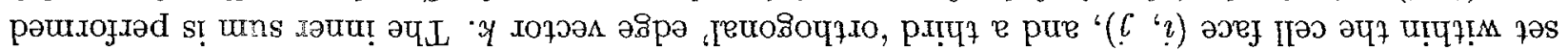

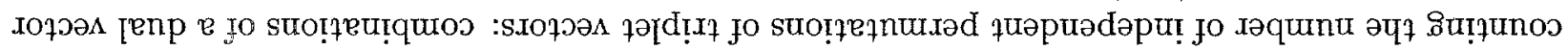

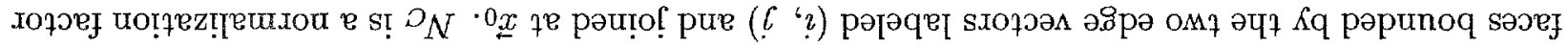

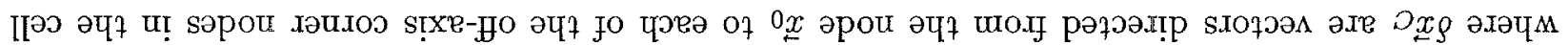
$\left(89^{\circ} \mathcal{\varepsilon}\right)$

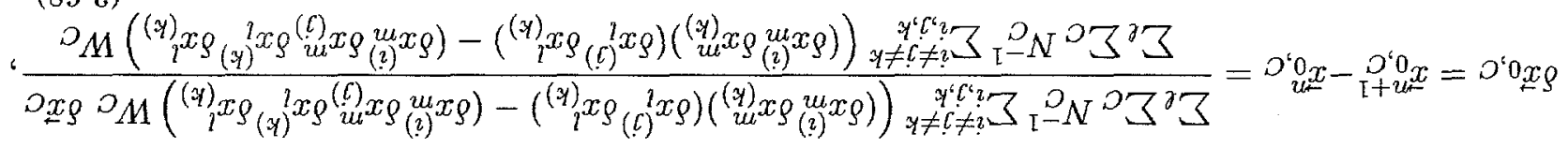

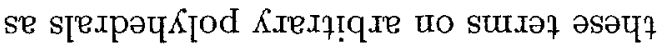

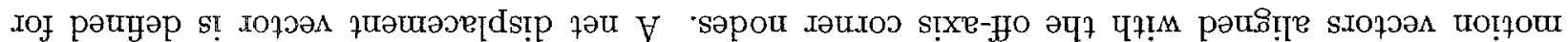

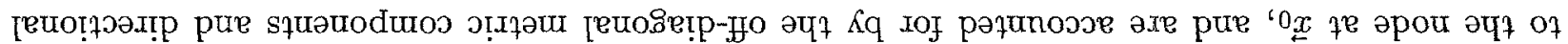

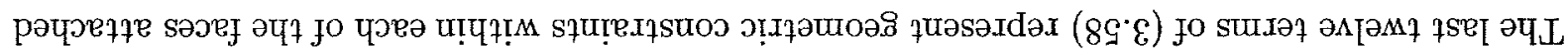

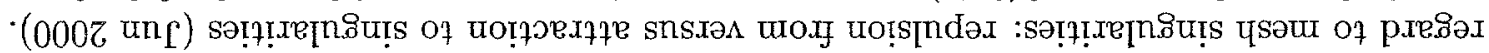

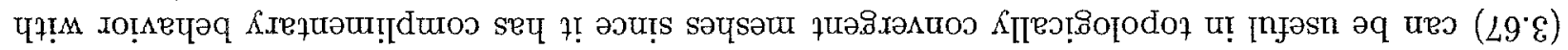

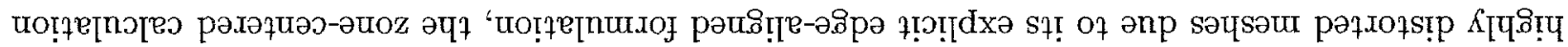

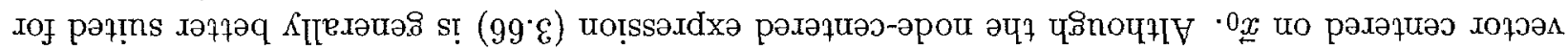
ә.8ิрә әч7 s!

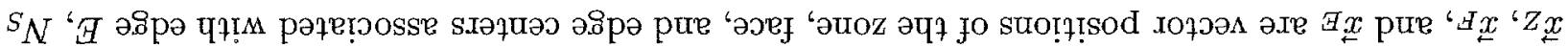
$\left(29^{\circ} \varepsilon\right)$

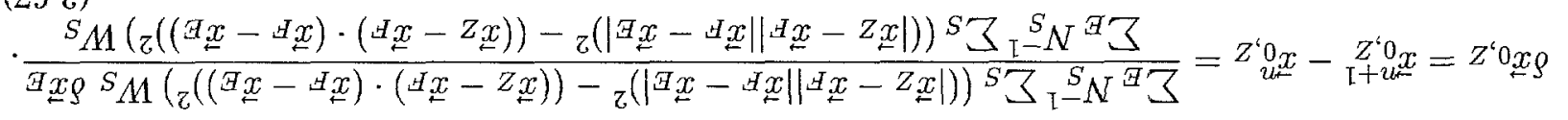

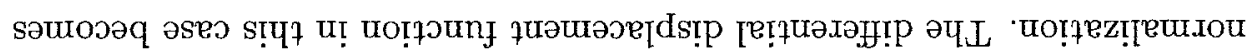

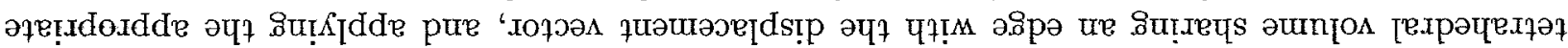

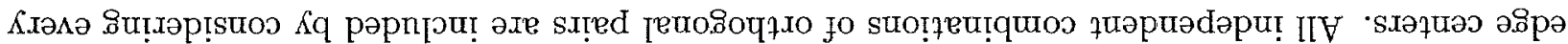

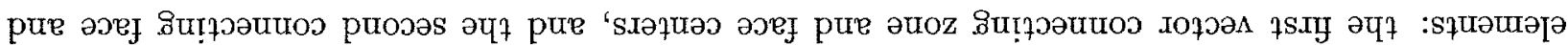

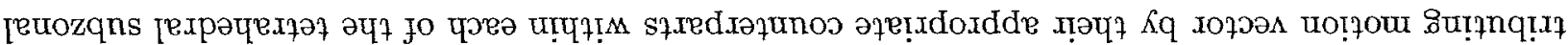

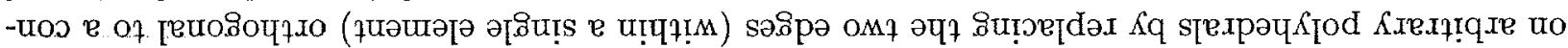

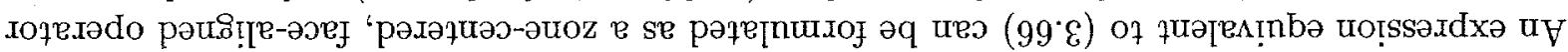

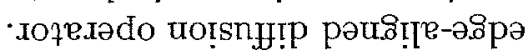

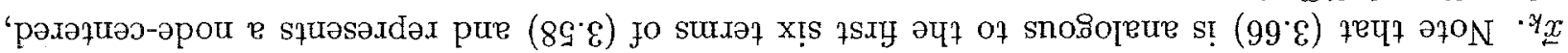

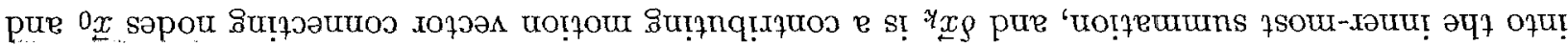

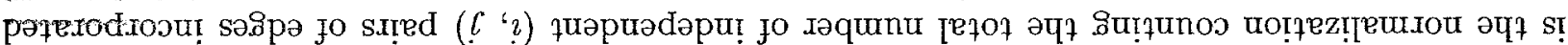

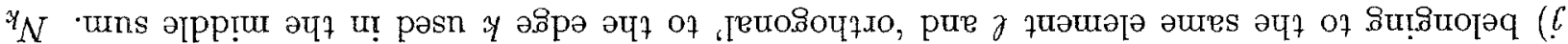

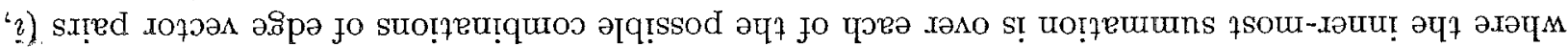
$\left(99^{\circ} \varepsilon\right)$

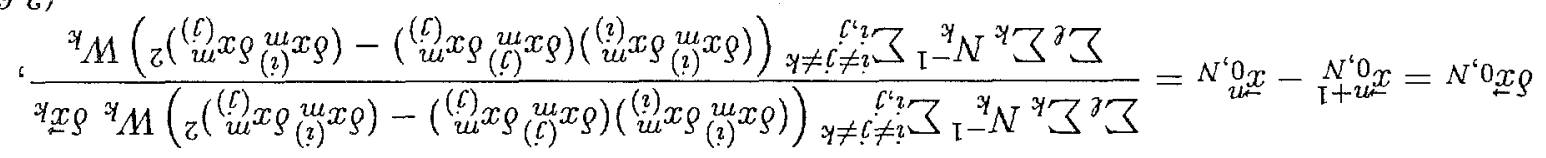

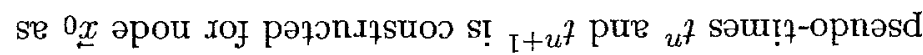


and the various weights appearing in (3.66) - (3.68) are tied to local cell attributes. In particular, $W=V^{w_{v}} E^{w_{e}}$, where $V$ is the cell (or tetrahedral sub-element) volume, $E$ is the edgelength, and $w_{v}$ and $w_{e}$ are arbitrary exponents.

\section{Post-motion filters}

Several constraints are placed on the final mesh displacement vector once it's computed by any of the methods described in sections $\S 1$ - 3. First, to prevent excessive diffusion errors in the ale remap cycle, the mesh motion is rescaled in amplitude to small relative changes in cell dimensions as $\delta \vec{x}_{0} \rightarrow \epsilon \delta \vec{x}_{0}$ with

$$
\epsilon=\min \left(1, \frac{C_{L} \Delta L}{\left|\delta \vec{x}_{0}\right|}, \frac{C_{V}}{\left|\nabla \cdot \delta \vec{x}_{0}\right|}, \max \left(0, \frac{\left|\delta \vec{x}_{0}\right|-\delta x_{\min }}{|| \delta \vec{x}_{0}\left|-\delta x_{\min }\right|}\right)\right)
$$

where $\Delta L$ is a characteristic length scale, $C_{L}$ and $C_{V}$ are constants with values less than unity constraining the relative length and volume changes respectively, and $\delta x_{\min }$ is a minimum displacement threshold below which any computed mesh motion is neglected.

Second, the displacement vector may be rescaled to the fluid velocity to prevent unnecessary mesh movement in domains which have not become dynamically active. This is achieved by setting

$$
\delta \vec{x}_{0} \rightarrow \delta \vec{x}_{0} \min \left(1, \frac{k_{1}\left|\vec{v}_{f}\right| \Delta t}{\left|\delta \vec{x}_{0}\right|}\right)
$$

where $k_{1}$ is an arbitrary scale constant, $\Delta t$ the physical Lagrange cycle timestep, and $\vec{v}_{f}$ the fluid velocity.

Finally, additional node and zone coupling can be achieved by applying local filters and effectively generating smoother meshes by extending the Laplacian stencil to larger domains of influence, and suppressing further any potential hourglassing modes. For example, the displacement vector may be modified to the form

$$
\delta \vec{x}_{0} \rightarrow \delta \vec{x}_{0}+k_{2}\left(\delta \vec{x}_{0, a v g}-\delta \vec{x}_{0}\right)
$$

where $k_{2}$ is a constant less than or equal to unity. $\delta \vec{x}_{0, a v g}$ is an extended average nodal mesh velocity computed either as a simple average of all node velocities directly connected to $\vec{x}_{0}$ by cell edges, or as a zone-centered extrapolated mean field. In the latter case, a zone-centered velocity $\delta \vec{x}_{0, Z}$ is computed in all zones attached to node 0 by averaging it's nearest nodal velocities; node-centered gradients of each of the zone-centered field components $\vec{\nabla}_{N} \delta x_{0, Z}^{(i)}$ are constructed and averaged back to the zone centers; $\delta \vec{x}_{0, a v g}$ is finally defined by extrapolating the nearest zone-centered velocities to node 0 using a first order Taylor's series to the form

$\delta \vec{x}_{0, a v g}=\frac{1}{N_{0, Z}} \sum_{m_{Z}=1}^{N_{0, Z}}\left(\delta \vec{x}_{0, m_{Z}}+\frac{1}{N_{N, Z}} \sum_{n=1}^{N_{N, Z}}\left(\begin{array}{ccc}\nabla_{n}^{(1)} \delta x_{0, Z}^{(1)} & \nabla_{n}^{(2)} \delta x_{0, Z}^{(1)} & \nabla_{n}^{(3)} \delta x_{0, Z}^{(1)} \\ \nabla_{n}^{(1)} \delta x_{0, Z}^{(2)} & \nabla_{n}^{(2)} \delta x_{0, Z}^{(2)} & \nabla_{n}^{(3)} \delta x_{0, Z}^{(2)} \\ \nabla_{n}^{(1)} \delta x_{0, Z}^{(3)} & \nabla_{n}^{(2)} \delta x_{0, Z}^{(3)} & \nabla_{n}^{(3)} \delta x_{0, Z}^{(3)}\end{array}\right)\left(\begin{array}{c}x_{0}^{(1)}-x_{0, m_{Z}}^{(1)} \\ x_{0}^{(2)}-x_{0, m_{Z}}^{(2)} \\ x_{0}^{(3)}-x_{0, m_{Z}}^{(3)}\end{array}\right)\right)$.

$N_{0, Z}$ is the total number of zones attached to node $0, N_{N, Z}$ the total number of nodes associated with zone $Z$, and $x_{0}^{i}-x_{0, m_{Z}}^{i}$ are the vector components of the distances between node 0 and nearest neighbor zone centers identified by the index $m_{Z}$. 
Acknowledgements This work was performed under the auspices of the U.S. Department of Energy by University of California, Lawrence Livermore National Laboratory under Contract W7405-Eng-48.

\section{References}

[1] A. Liu and B. Joe, BIT, 34, 268 (1994)

[2] L. Freitag and P. Knupp, 8th International Meshing RoundTable, Lake Tahoe NV (1999)

[3] W.P. Crowley, Technical Report, Lawrence Livermore National Laboratory (1962)

[4] A.M. Winslow, LLNL Report UCRL-7312 (1963)

[5] R. Tipton, Technical Report, Lawrence Livermore National Laboratory (1994)

[6] B.I. Jun, Technical Report, Lawrence Livermore National Laboratory (2000) 